A. Scott Nielsen, MD, MMSc

Revere P. Kinkel, MD

Nancy Madigan, PhD

Emanuele Tinelli, MD

Thomas Benner, PhD

Caterina Mainero, MD, $\mathrm{PhD}$

Correspondence to

Dr. Mainero:

caterina@nmr.mgh.harvard.edu

\title{
Contribution of cortical lesion subtypes at 7T MRI to physical and cognitive performance in $\mathrm{MS}$
}

ABSTRACT

Objectives: Evaluate cross-sectionally the contribution of focal cortical lesion (CL) subtypes at ultra-high-field MRI and traditional MRI metrics of brain damage to neurologic disability and cognitive performance in a heterogeneous multiple sclerosis (MS) cohort.

Methods: Thirty-four patients with early or established disease including clinically isolated syndrome, relapsing-remitting MS, and secondary progressive MS were scanned on a human 7-tesla (7T) (Siemens) scanner to acquire fast low-angle shot (FLASH) T2*-weighted images for characterization of white matter and deep gray matter lesion volume, and CL types. Patients also underwent anatomical 3T MRI for cortical thickness estimation, and neuropsychological testing within 1 week of the 7T scan. Twenty-seven patient scans were acceptable for further analysis. Neurologic disability was measured using the Expanded Disability Status Scale.

Results: Type III-IV CLs had the strongest relationship to physical disability $(\rho=0.670, p<0.0001)$. White matter lesion volume and type I CLs are each significantly associated with 6 of 11 neuropsychological test variables. Type III-IV CLs significantly correlate with 4 of 11 neuropsychological test variables whereas type II CLs, deep gray matter lesion volume, and cortical thickness metrics are less frequently associated with cognitive performance.

Conclusions: Leukocortical (type I) and subpial (III-IV) CLs identified on 7T FLASH-T2* sequences are potential cortical biomarkers of cognitive and neurologic status in MS. Neurology ${ }^{\circledR} 2013 ; 81: 641-649$

\section{GLOSSARY}

ANT = Animal Naming Test; BDI = Beck Depression Inventory, 2nd edition; BVMTR = Brief Visual Memory Test-Revised; $\mathbf{C L}=$ cortical lesion; $\mathbf{C L R}=$ cortical lesion ratio; $\mathbf{C O W A T}=$ Controlled Oral Word Association Test; $\mathbf{C T}=$ cortical thickness; CVLT2 = California Verbal Learning Test, 2 nd edition; DGMLV = deep gray matter lesion volume; DIR = double inversion recovery; EDSS = Expanded Disability Status Scale; FLASH = fast low-angle shot; $\mathbf{G M}=$ gray matter; JLOT = Judgment of Line Orientation Test; MEMPR = magnetization-prepared rapid acquisition with multiple gradient echoes; $\mathbf{M S}=$ multiple sclerosis; NPT = neuropsychological test; SDMT = Symbol Digit Modalities Test; $\mathbf{S P M S}=$ secondary progressive multiple sclerosis; $\mathbf{W C S T}=$ Wisconsin Card Sorting Test-64 card deck; WM $=$ white matter; $\mathbf{W M L V}=$ white matter lesion volume; WTAR $=$ Wechsler Test of Adult Reading.

Cognitive dysfunction is a common but frequently overlooked clinical manifestation of multiple sclerosis (MS). ${ }^{1}$ Frequently affected cognitive domains include executive function, processing speed, and memory. ${ }^{2}$ Cognitive impairment in MS deleteriously affects social and vocational activities, may result in unemployment, and appears to lead to an increased vulnerability to psychiatric illness. ${ }^{3}$ Despite the impact of cognitive dysfunction in MS, our understanding of its pathophysiology is limited.

Conventional and quantitative MRI have identified potential biomarkers and better defined the pathophysiology of cognitive dysfunction in MS. ${ }^{4}$ To date, global rather than regional metrics of cortical atrophy and cortical lesion (CL) volume using double inversion recovery (DIR) sequences at 3 tesla (3T) better account for the variance seen in cognitive performance in MS - supplanting conventional white matter (WM) MRI metrics., ${ }^{5,6}$ These findings are not entirely unexpected because MS neuropathology has highlighted the cortex as a major location of demyelination. ${ }^{7}$ Although 3T DIR is an innovative improvement over conventional imaging

From Virginia Mason Medical Center (A.S.N.), Seattle; University of Washington (A.S.N.), Seattle, WA; Beth Israel Deaconess Medical Center (R.P.K., N.M.), Boston; Harvard Medical School (R.P.K., N.M., C.M.), Boston, MA; Department of Neurology and Psychiatry (E.T.), University of Rome La Sapienza, Italy; and Athinoula A. Martinos Center for Biomedical Imaging (T.B., C.M.), Massachusetts General Hospital, Boston, MA. Go to Neurology.org for full disclosures. Funding information and disclosures deemed relevant by the authors, if any, are provided at the end of the article.
Supplemental data at www.neurology.org 
of cortical demyelination, ultra-high-field (7T) MRI using an optimized fast low-angle shot (FLASH)-T2* sequence detects more than 5 to 7 times the number of in vivo CLs differentiated by neuropathologic type-allowing identification of subpial lesions not easily detectable on DIR imaging, and which are thought to be associated with disease progression. ${ }^{8}$ Postmortem histopathologic-MRI correlations ${ }^{9}$ demonstrated an excellent $93 \%$ retrospective sensitivity of ex vivo focal CL detection using 7T MRI. Our purpose was to evaluate the relationship and contribution of individual global MRI metrics focused on the cortex, including neuropathologically defined focal CL types such as subpial, leukocortical, and purely intracortical CLs at 7T, with cognitive performance and physical disability status in MS.

METHODS Patient population. Thirty-four patients from the Beth Israel Deaconess MS Center participated in this study. Eligibility criteria included age 18 to 60 years and a diagnosis of clinically isolated syndrome or MS. ${ }^{10,11}$ Patients were recruited according to 3 disease phenotypic categories defined as follows: 1) clinically isolated syndrome/early MS $=<3$ years' disease duration and Expanded Disability Status Scale (EDSS) ${ }^{12}$ score $\leq 2 ; 2$ ) relapsing-remitting $\mathrm{MS}=>3$ years' disease duration and EDSS score $\geq 2 ; 3$ ) secondary progressive MS $=$ EDSS score $>3$. Exclusion criteria include a clinical relapse within 3 months of enrollment, corticosteroid therapy within 1 month of scanning, other neurologic and/or significant psychiatric disease, major medical comorbidity, pregnancy, and general contraindications for MRI. Individuals with significant visual or upper-extremity deficits that would interfere with the neuropsychological testing were also excluded. A board- and EDSS-certified neurologist (A.S.N.) performed the EDSS neurologic disability assessments.

Figure Subpial and leukocortical lesions in multiple sclerosis by ultra-high-field MRI
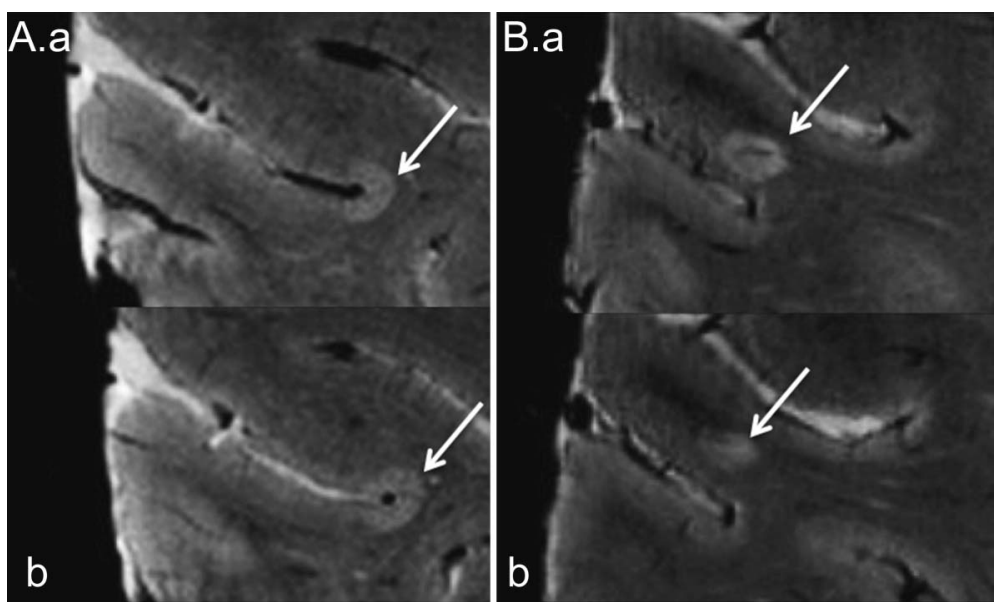

Axial FLASH-T2* brain images at 7T of a patient with multiple sclerosis. Consecutive slices demonstrate classic subpial (type III-IV; A.a and b) and leukocortical (type I; B.a and b) lesions (arrows). FLASH = fast low-angle shot; $T=$ tesla.
Neuropsychological evaluation. A neuropsychologist (N.M.) evaluated all patients within 1 week of the 7T MRI scan. Neuropsychological tests included the California Verbal Learning Test, 2nd edition (CVLT2), ${ }^{13}$ Brief Visual Memory Test-Revised (BVMTR), ${ }^{14}$ Wisconsin Card Sorting Test-64 card deck (WCST), ${ }^{15}$ Symbol Digit Modalities Test (SDMT), ${ }^{16}$ Judgment of Line Orientation Test-odd items (JLOT), ${ }^{17}$ Animal Naming Test (ANT), ${ }^{18}$ and Controlled Oral Word Association Test (COWAT). ${ }^{19}$ Premorbid intelligence was assessed using the Wechsler Test of Adult Reading (WTAR). ${ }^{20}$ The level of mood disturbance was estimated by the Beck Depression Inventory, 2nd edition (BDI) raw score. ${ }^{21}$

MRI. Acquisition. Subjects underwent T2* imaging on a $7 \mathrm{~T}$ Siemens MRI scanner (Siemens, Erlangen, Germany) for CL identification, using an in-house 32-channel, phased array coil. At the beginning of the $7 \mathrm{~T}$ scan, manual $\mathrm{B}_{0}$ shimming was performed to minimize susceptibility effects. The $7 \mathrm{~T}$ protocol included 2-dimensional FLASH-T2* ${ }^{*}$-weighted spoiled gradientecho images to cover the supratentorial brain (repetition time/ echo time $=1,000 / 22$ milliseconds, flip angle $=55^{\circ}, 0.33 \times$ $0.33 \times 1 \mathrm{~mm}^{3}$ resolution, 2 slabs, time for acquisition for each slab $=$ approximately 8 minutes). For cortical thickness (CT) assessment, we acquired, on a separate session on a $3 \mathrm{~T}$ scanner (Siemens TIM Trio) using a 32-channel coil, a 3-dimensional magnetization-prepared rapid acquisition with multiple gradient echoes (MEMPR) sequence $\left(0.9 \times 0.9 \times 0.9 \mathrm{~mm}^{3}\right.$; inversion time $=1,200$ milliseconds; repetition time $=2,530$ milliseconds; flip angle $=7^{\circ}$; echo time $=1.7+\mathrm{n} .1 .88$ milliseconds where $\mathrm{n}=$ $0, \ldots, 3$; field of view $=230 \mathrm{~mm}$; bandwidth $=651 \mathrm{~Hz} / \mathrm{px})$. For image positioning and acquisition across participants and across scans, we used "Auto-Align" software. ${ }^{22}$

Assessment of CLs and WM and deep gray matter lesion volume. Two independent and experienced reviewers, blinded to patient demographic and clinical information, scored the $7 \mathrm{~T}$ FLASH-T2* scans of all subjects according to previously described focal CL typing ${ }^{23-25}$ : type I (spanning the gray matter [GM]/WM boundary), type II (purely intracortical with no involvement of the pial surface or GM/WM boundary), and type III-IV (involving the pial surface and varying depths through the cortex) (figure). CLs identified by consensus of both raters were carried forward for analysis.

Additional MRI metrics included WM lesion volume (WMLV) and deep GM lesion volume (DGMLV) $\left(\mathrm{mm}^{3}\right)$, which were assessed by a licensed radiologist (E.T.) on the 7T FLASH-T2* scans using a semiautomated contouring technique (Alice software; Hayden Solutions, Boulder, CO). We introduce a new metric, the $\mathrm{CL}$ ratio (CLR), which is defined as follows: (type I + type II)/(type III-IV). Because of patient motion, subsequent images were satisfactory for CT measurement in 32 patients and focal CL counts in 28 patients. Of the 34 patients, only 27 had all MRI metrics available for analysis (presented data only reflect these 27 patients).

CT measurement. Global mean CT ( $\mathrm{mm})$ was obtained in each subject using FreeSurfer (http://surfer.nmr.mgh.harvard. edu) on the 3T MEMPR according to a previously described, multistep, automated segmentation procedure ${ }^{26,27}$ that calculates the GM/WM border and the GM/CSF (pial) border in the 3-dimensional image volume. CT is measured as the distance between the GM/WM boundary and the pial surface.

Statistical methods. Descriptive statistics are presented for the entire cohort and by MS phenotype. Spearman correlation coefficient was used for associations between individual MRI metrics, in addition to EDSS and MRI metrics.

Multivariate linear regression was used to identify MRI metric associations with cognitive performance. The respective 
neuropsychological test (NPT) raw scores were appropriately transformed where indicated to meet model assumptions for normality and were designated as the dependent variable. In total, 11 NPT variables were analyzed (SDMT total; COWAT total; ANT total; CVLT2 total, short and long delayed free recall; BVMTR total; JLOT-odd items; WCST perseverations, errors, and perseverative errors). All MRI metrics described above were maintained as candidate predictors and transformed as appropriate to satisfy model assumptions. Age, education, premorbid intelligence (WTAR) and depression (BDI) raw scores were considered as candidate predictors if their Pearson correlation to the dependent variable demonstrated a $p$ value $\leq 0.15$. BDI raw score and education proved unrelated to all NPT variables for our cohort (table e-1 on the Neurology ${ }^{\circledR}$ Web site at www.neurology.org). To avoid overfitting the models, a sample size of 27 usable $7 \mathrm{~T}$ scans allows for a maximum of 2 potential predictors; therefore, each MRI metric was modeled separately while adjusting for the most statistically significant candidate predictor (i.e., WTAR or age). Partial correlation coefficients are presented for comparison. For all statistical tests, the $p$ value was set at 0.05 . All statistical analyses were completed in SAS version 9.3 (SAS Institute, Cary, NC).

Standard protocol approvals, registrations, and patient consents. The local ethics committee of our institutions approved the experimental procedures, and written informed consent was obtained from all study participants.

RESULTS Descriptive statistics. Demographic, MRI, and NPT metrics are presented in table 1. MS phenotypes of increasing chronicity demonstrate higher CL counts-particularly subpial (type III-IV) and leukocortical (type I). Subpial CL counts increase 3.3-fold between early MS and secondary progressive MS (SPMS) phenotypes (total lesion count $=77$ and 254, respectively) whereas leukocortical lesions disproportionately increase 5.8-fold (total lesion count $=28$ and 161, respectively) -an observation that is reflected in the CLR. The SPMS phenotype demonstrates lower CT and higher WMLV compared with earlier disease. Median NPT variable scores in processing speed (SDMT), language (COWAT, ANT), executive (WCST), and learning/ memory (CVLT2, BVMTR) cognitive domains are relatively worse in the SPMS subgroup. Visuospatial scores (JLOT) appear to worsen in a linear fashion between MS phenotypes with increasing chronicity.

Physical disability and MRI metric associations. Disability as measured by the EDSS was significantly associated with CL count, particularly type III-IV lesions as previously reported ${ }^{28,29}$; Spearman $\rho$ ( $p$ value) for EDSS and CL type association: total CL $=0.640(0.0003)$, type I CL $=0.449$ (0.02), type II CL $=0.188(0.35)$, type III-IV $=0.670(0.0001)$, and CLR $=0.181$ (0.39). This association held for CT and subcortical MRI metrics as well: EDSS and conventional MRI metric association: CT $=-0.655(0.0002)$ and WMLV $=0.389(0.05)$.

MRI metric associations. DGMLV is not significantly correlated with any MRI metric (table 2). All CL subtypes are associated with one another, with the most significant association observed between type I and III-IV CLs. Reduced CT is associated with increased type I and III-IV CL counts; however, the strongest association is seen with type III-IV lesions. Accumulation of WMLV is related to increased number of type I and III-IV CL subtypes, whereas type I CLs have the strongest correlation.

Regression analyses of MRI metrics to cognitive performance. Significant results are presented in table 3; all models are provided in table e- 2 . Because of necessary statistical transformations for both dependent and predictor variables, back transformation of the data for presentation is not practical. Therefore, caution with interpretation of the $\beta$ coefficients is necessary because they are not amenable to direct comparison within or between the presented models to assess the relative strength of association. However, partial correlation coefficients and adjusted model $R^{2}$ are provided for comparison of MRI metric predictors and their relative magnitude of association with cognitive performance.

A consistent strength of association is seen for global WMLV and type I CL counts with NPT variables reflecting cognitive performance in visuospatial, learning/memory, processing speed, and semantic (ANT) language domains. Both WMLV and type I CLs are most frequently associated with cognitive performance (6/11 variables, 55\%). Type III-IV CLs demonstrate a significant relationship with cognitive performance, although less frequently (4/11 variables, 36\%). Type III-IV CLs are associated with performance in learning/memory (with the exception of CVLT2 total) and semantic (ANT), but not phonemic (COWAT), language tests. In our cohort, infrequently associated MRI metrics with cognitive performance include CT (2/11 variables, 18\%), whereas type II CLs and DGMLV are significantly related to one variable each after adjustment by WTAR. Overall, the strength of association of WMLV and type I and III-IV CL metrics with cognitive performance are comparable (partial correlation coefficient range: -0.422 to $-0.602,-0.389$ to -0.622 , and -0.436 to -0.633 , respectively). Of the 3 NPT variables where WMLV and type I and type III-IV CL metrics remained significant after adjustment, all MRI metrics had comparable statistical association except for BVMTR. In this case, partial correlation coefficients favored type III-IV and type I CLs over WMLV $(-0.633,-0.622$, and -0.489 , respectively).

DISCUSSION To date, our cohort is the largest used to investigate the relationship between cognitive performance and physical disability and cortical and WM brain pathology in vivo using ultra-high-field (7T) MRI. Our findings are consistent with our 
Table 1 Demographic, MRI, and cognitive metrics

\begin{tabular}{|c|c|c|c|c|}
\hline & Total $(n=27)$ & CIS/early MS $(n=10)$ & RRMS ( $n=8)$ & SPMS (n = 9) \\
\hline \multicolumn{5}{|l|}{ Demographics } \\
\hline Age, $y$, mean $\pm S D$ & $43.0 \pm 7.9$ & $40.9 \pm 8.0$ & $40.9 \pm 8.3$ & $47.2 \pm 6.2$ \\
\hline Female, n (\%) & $19(70.4)$ & $7(70)$ & $6(75)$ & $6(67)$ \\
\hline MS duration, $y$, mean \pm SD & $9.3 \pm 7.3$ & $2.0 \pm 0.7$ & $10.4 \pm 4.2$ & $16.4 \pm 5.6$ \\
\hline EDSS score, median (range) & $3(0-6)$ & $1.5(0-2)$ & $3.0(2-4)$ & $4.5(3-6)$ \\
\hline Education, y, median (range) & $16(12-20)$ & $16(12-19)$ & $16(12-18)$ & $16(12-20)$ \\
\hline \multicolumn{5}{|l|}{ MRI } \\
\hline Total CL count ${ }^{\mathrm{a}}$ & $20(0-85)$ & $9.5(0-37)$ & $18.0(1-34)$ & $43.0(20-85)$ \\
\hline Type I & $5.0(0-42)$ & $1.0(0-15)$ & $4.5(0-22)$ & $15.0(1-42)$ \\
\hline Type II & $0(0-2)$ & $0(0-2)$ & $0(0-0)$ & $0(0-2)$ \\
\hline Type III-IV & $14.0(0-41)$ & $7.0(0-20)$ & $12.0(1-29)$ & $29.0(14-41)$ \\
\hline $\mathrm{CLR}_{r}^{\mathrm{b}}$ median (range) & $0.25(0-2)$ & $0.11(0-1)$ & $0.24(0-2)$ & $0.70(0.03-1.24)$ \\
\hline $\mathrm{CT}$, mm, median (range) & $2.54(1.93-2.89)$ & $2.61(2.54-2.89)$ & $2.56(2.34-2.77)$ & $2.43(1.93-2.57)$ \\
\hline WMLV, mm ${ }^{3}$, median (range) & $1,270.0(64-15,718)$ & $363.2(82-3,176)$ & $285.1(64-10,616)$ & $5,623(1,034-15,718)$ \\
\hline DGMLV, mm³, median (range) & $71.3(2.7-257.6)$ & $89.4(30.5-257.6)$ & $55.4(24.7-170.2)$ & $55(2.7-200.8)$ \\
\hline \multicolumn{5}{|l|}{ Cognitive testing, median (range) } \\
\hline $\mathrm{SDMT}_{\text {total }}$ & $61.0(32-73)$ & $63.5(38-73)$ & $65.0(49-72)$ & $41.0(32-69)$ \\
\hline $\operatorname{COWAT}_{\text {total }}$ & $45.0(12-78)$ & 45.5 (25-78) & $47.5(27-65)$ & $38.0(12-47)$ \\
\hline $\mathrm{ANT}_{\text {total }}$ & $24.0(11-38)$ & $25.0(14-38)$ & $25.0(18-38)$ & $19.0(11-24)$ \\
\hline CVLT2 $2_{\text {total }}$ & $54.0(31-75)$ & $55.0(45-75)$ & $61.5(51-68)$ & $44.0(31-66)$ \\
\hline CVLT2 & $12.0(6-16)$ & $12.5(10-16)$ & $13.5(10-16)$ & $9.0(6-16)$ \\
\hline CVLT2 ${ }_{\text {LDFR }}$ & $13.0(5-16)$ & $15.0(7-16)$ & $14.0(10-16)$ & $10.0(5-15)$ \\
\hline BVMTR $_{\text {total }}$ & $28.0(10-35)$ & $30.0(24-35)$ & $29.5(22-33)$ & $19.0(10-33)$ \\
\hline $\mathrm{JLOT}_{\text {odd }}$ & $12.0(5-15)$ & $14.0(11-15)$ & $12.0(8-15)$ & $10.0(5-13)$ \\
\hline WCST $_{\text {perseverations }}$ & 7.0 (3-22) & $5.0(3-15)$ & $7.0(4-20)$ & $12.0(3-22)$ \\
\hline WCST $_{\text {errors }}$ & $12.0(5-37)$ & $9.0(5-28)$ & $11.5(5-25)$ & $20.0(8-37)$ \\
\hline WCST $_{\text {perseverative errors }}$ & $7.0(3-19)$ & $5.0(3-14)$ & $6.5(4-14)$ & $10.0(3-19)$ \\
\hline WTAR $_{\text {raw }}$ & $43.0(13-50)$ & $46.5(32-50)$ & $42.0(28-49)$ & $38.0(13-46)$ \\
\hline BDI & $6.0(0-26)$ & $5.5(0-11)$ & $6.0(0-26)$ & $11.0(0-21)$ \\
\hline
\end{tabular}

Abbreviations: ANT = Animal Naming Test; BDI = Beck Depression Inventory, 2nd edition; BVMTR = Brief Visual Memory Test-Revised; CIS = clinically isolated syndrome; $\mathrm{CL}=$ cortical lesion; $\mathrm{CLR}=$ cortical lesion ratio; COWAT = Controlled Oral Word Association Test; $\mathrm{CT}=$ cortical thickness; CVLT2 = California Verbal Learning Test, 2nd edition; DGMLV = deep gray matter lesion volume; EDSS = Expanded Disability Status Scale; JLOT = Judgment of Line Orientation Test; LDFR = long delayed free recall; MS = multiple sclerosis; RRMS = relapsing-remitting multiple sclerosis; SDFR = short delayed free recall; SDMT = Symbol Digit Modalities Test; SPMS = secondary progressive multiple sclerosis; WCST = Wisconsin Card Sorting Task-64 deck; WMLV = white matter lesion volume; WTAR $=$ Wechsler Test of Adult Reading.

${ }^{a}$ Median (range) per group.

${ }^{\mathrm{b}} \mathrm{CLR}=($ type I + type II)/(type III-IV).

previous report demonstrating a strong association between subpial (type III-IV) CL type and physical disability (EDSS). ${ }^{28}$ This is consistent with prior neuropathology literature, which reported the association of subpial cortical demyelination and shorter time to wheelchair status. ${ }^{29,30}$ Conventional-strength MRI $(\leq 3 \mathrm{~T})$ is relatively insensitive in detecting subpial demyelination and almost exclusively identifies leukocortical (type I) lesions. ${ }^{8,31}$ Alternatively, optimized T2* sequences at 7T MRI demonstrate the ability to detect all neuropathologically defined CL types in vivo with similar frequency, and ex vivo validation has shown excellent retrospective sensitivity of $93 \%$ for cortical demyelination at 7T, ${ }^{9,28}$

Among our cohort, several measures of cognitive performance were significantly associated with global WMLV and type I CL counts. The relationship between these 2 MRI metrics is quite strong and statistically significant (table 2). This observation is likely attributable to the fact that type I CLs by definition involve both the GM and WM. What remains unknown is the relative contribution of WM 


\begin{tabular}{|c|c|c|c|c|c|c|c|}
\hline \multirow[t]{2}{*}{ Table 2} & \multicolumn{7}{|c|}{ MRI metric correlations $(n=27)^{a}$} \\
\hline & WMLV & DGMLV & $\mathrm{CT}$ & Total CL & Type I & Type II & Type III \\
\hline DGMLV & $-0.087(0.67)$ & - & - & - & - & - & - \\
\hline Ст & $-0.219(0.27)$ & $0.189(0.35)$ & - & - & - & - & - \\
\hline Total CL & $0.694(<0.0001)^{b}$ & $-0.148(0.46)$ & $-0.526(0.005)^{b}$ & - & - & - & - \\
\hline Type I CL & $0.803(<0.0001)^{b}$ & $0.089(0.66)$ & $-0.406(0.04)^{b}$ & $0.889(<0.0001)^{b}$ & - & - & - \\
\hline Type II CL & $0.324(0.10)$ & $0.238(0.23)$ & $-0.108(0.59)$ & $0.509(0.007)^{b}$ & $0.529(0.005)^{b}$ & - & - \\
\hline Type III-IV CL & $0.578(0.002)^{b}$ & $-0.223(0.26)$ & $-0.591(0.001)^{\mathrm{b}}$ & $0.960(<0.0001)^{b}$ & $0.760(<0.0001)^{b}$ & $0.419(0.03)^{b}$ & - \\
\hline CLR & $0.711(<0.0001)^{\mathrm{b}}$ & $0.216(0.30)$ & $-0.236(0.26)$ & $0.629(0.0008)^{b}$ & $0.910(<0.0001)^{\mathrm{b}}$ & $0.485(0.01)^{b}$ & $0.406(0.04)^{\mathrm{b}}$ \\
\hline
\end{tabular}

Abbreviations: $\mathrm{CL}=$ cortical lesion; $\mathrm{CLR}=\mathrm{CL}$ ratio; $\mathrm{CT}=$ cortical thickness; $\mathrm{DGMLV}=$ deep gray matter lesion volume; WMLV $=$ white matter lesion volume.

${ }^{a}$ Data represent Spearman $\rho$ ( $p$ value).

b Significant value.

involvement from type I CLs to cognitive performance vs that of deeper WM pathology, which is also reflected in the WMLV metric. Our cohort sample size does not support a multivariate regression model to determine which, if any, of the presented MRI metrics at $7 \mathrm{~T}$ are independent predictors of cognitive performance.

A prior study of a larger cohort $(\mathrm{n}=70)$ with a comparable number of MRI predictors reported that CL count and volume, and normalized brain and cortical volume, were independent MRI metric predictors of cognitive performance using 3T DIR. ${ }^{6} \mathrm{We}$ recently published data indicating that 3 T DIR sequences have a predicted sensitivity of $13.6 \%$ to $18.3 \%$ for $\mathrm{CL}$ detection compared with $7 \mathrm{~T}$ FLASH-T2* as a reference standard. ${ }^{8}$ Although no subpial CLs were detected using $3 \mathrm{~T}$ DIR, type I lesions were the most frequently detected $\mathrm{CL}$ using this technique. ${ }^{8}$ Assuming their cohort' ${ }^{6}{ }^{6} \mathrm{CL}$ count reflects type I CLs, such an observation is congruent with our current observation that type I CLs demonstrate comparable, and at times, greater, magnitude of association with cognitive performance than other MRI metrics at 7T.

Our data suggest that WMLV and CL countsparticularly type I and III-IV_-have comparable ability to account for the variance seen in cognitive performance. The statistically significant association between WMLV and cognition may be a reflection of functional disruption of relevant cortical-subcortical circuits subserving cognition in addition to improved sensitivity of WM pathology detection afforded by 7T relative to conventional-strength MRI. In addition to WMLV, type I and III-IV CLs are more frequently retained in the regression models as significant predictors of cognitive performance than global CT and DGMLV metrics at $7 \mathrm{~T}$. The moderate adjusted model $R^{2}$ (ranging from $20 \%$ to $49 \%$ ) from our study suggests that future studies with a combination of demographic, patient self-report, and imaging metrics_-including other novel sequences such as diffusion tensor imaging ${ }^{32}$ will likely provide a more comprehensive understanding of cognitive dysfunction in MS. In addition, we performed analyses of cognitive performance according to cognitive domains and as "impaired" vs "unimpaired" cognitive status (see tables e-3 and e- 4 ). The results are congruent with the presented raw data in that WMLV and type I and III-IV CLs are significantly associated and share a similar strength of association with cognitive performance in MS. Our preliminary observations suggest that WMLV and subpial (type III-IV) and leukocortical (type I) CLs may be potential biomarkers of cognitive dysfunction in MS. Further validation with a larger cohort is needed.

The frequent association between type I CLs and cognitive dysfunction in our cohort may reflect a greater effect of this CL subtype on disruption of subcortical U-fibers and regional cortical-to-cortical communication, which is necessary for efficient cognitive functioning. Indeed, previous data ${ }^{33}$ on $3 \mathrm{~T}$ DIR demonstrated that "mixed" CLs_-defined as primarily located in the cortex with approximately $25 \%$ lesion extension into the WM-had the greatest statistical association with cognitive impairment. Their "mixed" CL is congruent with our present definition of type I CL, and further validates the potential role of this CL subtype in cognitive dysfunction observed in MS. Future investigation can test this hypothesis, and determine whether accumulation of type I CLs over time in a longitudinal sample may reach a threshold in which brain plasticity can no longer compensate, resulting in identifiable cognitive impairment.

Our cross-sectional data trends suggest that earlier MS demonstrates proportionately fewer type I CLs than type III-IV lesions when compared with relapsing-remitting MS and SPMS (table 1). This finding coupled with the observation of type III-IV CLs from the earliest disease stages is supportive of the "outside-in" hypothesis whereby cortical demyelination is initiated 
Table 3 Significant regression analyses of candidate MRI metric predictors to neuropsychological testing performance

MRI metric associations to SDMT total (age-adjusted)

\begin{tabular}{|c|c|c|c|c|c|c|c|c|}
\hline & $\rho(p)^{a}$ & $R\left[95 \% \mathrm{Cl}^{\mathrm{b}}\right.$ & +Intercept & & & $+\mathrm{Age}^{\mathrm{c}}$ & & \\
\hline & & & $\beta(95 \% \mathrm{Cl})$ & p & $\operatorname{adj} R^{2}$ & $\beta(95 \% \mathrm{Cl})$ & p & $\operatorname{adj}^{2}$ \\
\hline WMLV $^{d}$ & $-0.447(0.02)^{\mathrm{e}}$ & $-0.422[-0.691,-0.033]^{\mathrm{e}}$ & $-3.66(-6.67,-0.64)^{e}$ & $0.02^{\mathrm{e}}$ & $0.17^{\mathrm{e}}$ & $-3.31(-6.31,-0.31)^{e}$ & $0.03^{\mathrm{e}}$ & $0.20^{\mathrm{e}}$ \\
\hline Type $I^{c}$ & $-0.544(0.003)^{e}$ & $-0.471[-0.721,-0.093]^{\mathrm{e}}$ & $-3.81(-6.22,-1.39)^{e}$ & $<0.01^{\mathrm{e}}$ & $0.20^{\mathrm{e}}$ & $-3.52(-6.29,-0.74)^{e}$ & $0.02^{\mathrm{e}}$ & $0.24^{e}$ \\
\hline \multicolumn{9}{|c|}{ MRI metric associations to $A N T_{\text {total }}$ (WTAR-adjusted) } \\
\hline & $\rho(p)^{a}$ & $R[95 \% \mathrm{Cl}]^{\mathrm{b}}$ & +Intercept & & & +WTAR & & \\
\hline & & & $\beta(95 \% \mathrm{Cl})$ & $p$ & $\operatorname{adj} R^{2}$ & $\beta(95 \% \mathrm{Cl})$ & $p$ & $\operatorname{adj} R^{2}$ \\
\hline WMLV $^{d}$ & $-0.589(0.001)^{e}$ & $-0.515[-0.748,-0.150]$ & $-2.61(-4.09,-1.13)^{\mathrm{e}}$ & $0.001^{\mathrm{e}}$ & $0.32^{e}$ & $-2.36(-4.01,-0.71)^{\mathrm{e}}$ & $0.007^{e}$ & $0.31^{\mathrm{e}}$ \\
\hline DGMLV $^{c}$ & $-0.266(0.18)$ & $-0.415[-0.687,-0.024]^{\mathrm{e}}$ & $-0.54(-1.36,0.27)$ & 0.18 & 0.03 & $-0.83(-1.59,-0.06)^{\mathrm{e}}$ & $0.04^{\mathrm{e}}$ & $0.22^{e}$ \\
\hline $\mathrm{CT}$ & $0.479(0.01)^{\mathrm{e}}$ & $0.481[0.105,0.727]^{\mathrm{e}}$ & $20.00(4.91,35.08)^{e}$ & $0.01^{e}$ & $0.20^{\mathrm{e}}$ & $18.78(4.35,33.22)^{e}$ & $0.01^{\mathrm{e}}$ & $0.28^{e}$ \\
\hline Total $\mathrm{CL}^{\mathrm{c}}$ & $-0.407(0.04)^{\mathrm{e}}$ & $-0.442[-0.704,-0.057]^{\mathrm{e}}$ & $-1.24(-2.38,-0.09)^{e}$ & $0.04^{\mathrm{e}}$ & $0.13^{e}$ & $-1.25(-2.33,-0.18)^{\mathrm{e}}$ & $0.02^{\mathrm{e}}$ & $0.24^{e}$ \\
\hline Type I & $-0.522(0.005)^{\mathrm{e}}$ & $-0.515[-0.748,-0.149]^{\mathrm{e}}$ & $-1.98(-3.31,-0.65)^{\mathrm{e}}$ & $0.005^{e}$ & $0.24^{\mathrm{e}}$ & $-1.84(-3.12,-0.55)^{\mathrm{e}}$ & $0.007^{e}$ & $0.31^{\mathrm{e}}$ \\
\hline Type III-IV & $-0.383(0.049)^{\mathrm{e}}$ & $-0.436[-0.700,-0.050]^{\mathrm{e}}$ & $-0.23(-0.45,-0.001)^{\mathrm{e}}$ & $0.049^{e}$ & $0.11^{e}$ & $-0.24(-0.45,-0.03)^{e}$ & $0.03^{\mathrm{e}}$ & $0.24^{e}$ \\
\hline \multicolumn{9}{|c|}{ MRI metric associations to JLOT odd (WTAR-adjusted) } \\
\hline & $\rho(p)^{a}$ & $R[95 \% \mathrm{Cl}]^{\mathrm{b}}$ & +Intercept & & & +WTAR & & \\
\hline & & & $\beta(95 \% \mathrm{Cl})$ & p & $\operatorname{adj} R^{2}$ & $\beta(95 \% \mathrm{Cl})$ & $p$ & $\operatorname{adj} R^{2}$ \\
\hline WMLV $^{d}$ & $-0.591(0.001)^{e}$ & $-0.524[-0.753,-0.161]^{\mathrm{e}}$ & $-0.84(-1.32,-0.37)^{e}$ & $0.001^{\mathrm{e}}$ & $0.32^{\mathrm{e}}$ & $-0.78(-1.31,-0.24)^{e}$ & $0.006^{\mathrm{e}}$ & $0.31^{\mathrm{e}}$ \\
\hline Total $\mathrm{CL}^{\mathrm{c}}$ & $-0.492(0.009)^{\mathrm{e}}$ & $-0.529[-0.756,-0.167]^{\mathrm{e}}$ & $-0.48(-0.83,-0.13)^{\mathrm{e}}$ & $0.009^{e}$ & $0.21^{e}$ & $-0.49(-0.81,-0.16)^{\mathrm{e}}$ & $0.006^{\mathrm{e}}$ & $0.31^{\mathrm{e}}$ \\
\hline Type $I^{c}$ & $-0.573(0.002)^{\mathrm{e}}$ & $-0.568[-0.779,-0.221]^{\mathrm{e}}$ & $-0.70(-1.11,-0.29)^{e}$ & $0.002^{e}$ & $0.30^{\mathrm{e}}$ & $-0.66(-1.06,-0.26)^{e}$ & $0.002^{\mathrm{e}}$ & $0.35^{\mathrm{e}}$ \\
\hline Type II $^{\mathrm{c}}$ & $-0.408(0.03)^{\mathrm{e}}$ & $-0.428[-0.695,-0.041]^{\mathrm{e}}$ & $-2.13(-4.09,-0.16)^{e}$ & $0.03^{\mathrm{e}}$ & $0.13^{\mathrm{e}}$ & $-2.10(-3.97,-0.23)^{\mathrm{e}}$ & $0.03^{\mathrm{e}}$ & $0.22^{\mathrm{e}}$ \\
\hline $\mathrm{CLR}^{\mathrm{c}}$ & $-0.536(0.006)^{e}$ & $-0.492[-0.743,-0.100]^{\mathrm{e}}$ & $-3.23(-5.42,-1.03)^{e}$ & $0.006^{e}$ & $0.26^{e}$ & $-2.78(-4.96,-0.61)^{e}$ & $0.01^{\mathrm{e}}$ & $0.32^{\mathrm{e}}$ \\
\hline \multicolumn{9}{|c|}{ MRI metric associations to CVLT2 $2_{\text {total }}$ (age-adjusted) } \\
\hline & $\rho(p)^{a}$ & $R(95 \% \mathrm{Cl})^{\mathrm{b}}$ & +Intercept & & & + Age $^{c}$ & & \\
\hline & & & $\beta(95 \% \mathrm{Cl})$ & p & $\operatorname{adj} R^{2}$ & $\beta(95 \% \mathrm{Cl})$ & $p$ & $\operatorname{adj} R^{2}$ \\
\hline WMLV $^{d}$ & $-0.542(0.004)^{e}$ & $-0.526[-0.754,-0.164]^{\mathrm{e}}$ & $-3.69(-6.05,-1.34)^{e}$ & $0.004^{e}$ & $0.27^{\mathrm{e}}$ & $-3.35(-5.63,-1.07)^{e}$ & $0.006^{e}$ & $0.33^{\mathrm{e}}$ \\
\hline \multicolumn{9}{|c|}{ MRI metric associations to CVLT2 $2_{\text {SDFR }}^{c}$ (WTAR-adjusted) } \\
\hline & $\rho(p)^{a}$ & $R[95 \% \mathrm{Cl}]^{\mathrm{b}}$ & +Intercept & & & +WTAR & & \\
\hline & & & $\beta(95 \% \mathrm{Cl})$ & $p$ & $\operatorname{adj} R^{2}$ & $\beta(95 \% \mathrm{Cl})$ & $p$ & $\operatorname{adj} R^{2}$ \\
\hline WMLV & $-0.677(0.0001)^{\mathrm{e}}$ & $-0.602[-0.798,-0.269]^{\mathrm{e}}$ & $-0.18(-0.27,-0.10)^{e}$ & $0.0001^{\mathrm{e}}$ & $0.44^{\mathrm{e}}$ & $-0.16(-0.25,-0.07)^{e}$ & $0.001^{\mathrm{e}}$ & $0.44^{e}$ \\
\hline Total $\mathrm{CL}^{\mathrm{c}}$ & $-0.492(0.009)^{e}$ & $-0.555[-0.771,-0.202]^{\mathrm{e}}$ & $-0.09(-0.16,-0.02)^{e}$ & $0.009^{e}$ & $0.21^{e}$ & $-0.09(-0.15,-0.03)^{\mathrm{e}}$ & $0.003^{\mathrm{e}}$ & $0.39^{e}$ \\
\hline Type Ic & $-0.553(0.003)^{\mathrm{e}}$ & $-0.558[-0.773,-0.207]^{\mathrm{e}}$ & $-0.13(-0.21,-0.05)^{e}$ & $0.003^{e}$ & $0.28^{e}$ & $-0.12(-0.19,-0.04)^{e}$ & $0.003^{e}$ & $0.40^{\mathrm{e}}$ \\
\hline
\end{tabular}




\section{Table 3 Continued}

MRI metric associations to $\mathrm{CVLT}_{\mathrm{SDFR}^{\mathrm{C}}}{ }^{\mathrm{C}}$ (WTAR-adjusted)

\begin{tabular}{|c|c|c|c|c|c|c|c|c|}
\hline Type III-IV & $-0.484(0.01)^{\mathrm{e}}$ & $-0.571[-0.780,-0.225]^{\mathrm{e}}$ & $-0.02(-0.03,-0.005)^{\mathrm{e}}$ & $0.01^{\mathrm{e}}$ & $0.20^{\circ}$ & $-0.02(-0.03,-0.01)^{\mathrm{e}}$ & $0.002^{\mathrm{e}}$ & $0.41^{\mathrm{e}}$ \\
\hline $\mathrm{CLR}^{\mathrm{c}}$ & $-0.461(0.02)^{e}$ & $-0.406[-0.691,-0.006]^{\mathrm{e}}$ & $-0.55(-1.01,-0.09)^{e}$ & $0.02^{\mathrm{e}}$ & $0.18^{\mathrm{e}}$ & $-0.45(-0.89,-0.002)^{\mathrm{e}}$ & $0.049^{e}$ & $0.26^{\mathrm{e}}$ \\
\hline
\end{tabular}

MRI metric associations to CVLT2 $_{\text {LDFR }}{ }^{\mathrm{C}}$ (WTAR-adjusted)

\begin{tabular}{|c|c|c|c|c|c|c|c|c|}
\hline & $\rho(p)^{\mathrm{a}}$ & $R[95 \% \mathrm{Cl}]^{\mathrm{b}}$ & +Intercept & & & +WTAR & & \\
\hline & & & $\beta(95 \% \mathrm{Cl})$ & p & $\operatorname{adj}^{2}$ & $\beta(95 \% \mathrm{Cl})$ & $p$ & $\operatorname{adj} R^{2}$ \\
\hline WMLV $^{d}$ & $-0.456(0.02)^{\mathrm{e}}$ & $-0.296[-0.609,0.109]$ & $-0.13(-0.24,-0.03)^{\mathrm{e}}$ & $0.02^{\mathrm{e}}$ & $0.18^{\mathrm{e}}$ & $-0.08(-0.19,0.03)$ & 0.14 & 0.30 \\
\hline Type $I^{c}$ & $-0.393(0.04)^{\mathrm{e}}$ & $-0.389[-0.670,-0.006]^{\mathrm{e}}$ & $-0.10(-0.19,-0.004)^{\mathrm{e}}$ & $0.04^{\mathrm{e}}$ & $0.12^{\mathrm{e}}$ & $-0.08(-0.17,-0.0001)^{\mathrm{e}}$ & $0.0497^{e}$ & $0.35^{\mathrm{e}}$ \\
\hline Type III-IV & $-0.359(0.07)$ & $-0.469[-0.720,-0.091]^{\mathrm{e}}$ & $-0.01(-0.03,0.001)$ & 0.07 & 0.09 & $-0.02(-0.03,-0.003)^{e}$ & $0.02^{\mathrm{e}}$ & $0.40^{\mathrm{e}}$ \\
\hline \multicolumn{9}{|c|}{ MRI metric associations to BVMTR $\mathrm{R}_{\text {total }}$ (WTAR-adjusted) } \\
\hline & $\rho(p)^{a}$ & $R\left[95 \% \mathrm{Cl}^{\mathrm{b}}\right.$ & +Intercept & & & + WTAR & & \\
\hline & & & $\beta(95 \% \mathrm{Cl})$ & $p$ & $\operatorname{adj}^{2}$ & $\beta(95 \% \mathrm{Cl})$ & $p$ & $\operatorname{adj} R^{2}$ \\
\hline WMLV $^{d}$ & $-0.580(0.002)^{\mathrm{e}}$ & $-0.489[-0.732,-0.116]^{\mathrm{e}}$ & $-2.44(-3.85,-1.03)^{\mathrm{e}}$ & $0.002^{\mathrm{e}}$ & $0.31^{\mathrm{e}}$ & $-2.06(-3.61,-0.51)^{\mathrm{e}}$ & $0.01^{\mathrm{e}}$ & $0.32^{\mathrm{e}}$ \\
\hline Ст & $0.487(0.01)^{e}$ & $0.497[0.126,0.737]^{e}$ & $19.28(5.04,33.51)^{\mathrm{e}}$ & $0.01^{\mathrm{e}}$ & $0.21^{\mathrm{e}}$ & $17.91(4.73,31.10)^{\mathrm{e}}$ & $0.01^{\mathrm{e}}$ & $0.33^{\mathrm{e}}$ \\
\hline Total $\mathrm{CL}^{\mathrm{c}}$ & $-0.584(0.001)^{\mathrm{e}}$ & $-0.650[-0.825,-0.340]^{\mathrm{e}}$ & $-1.68(-2.65,-0.72)^{\mathrm{e}}$ & $0.001^{e}$ & $0.32^{\mathrm{e}}$ & $-1.70(-2.54,-0.86)^{\mathrm{e}}$ & $0.0003^{\mathrm{e}}$ & $0.49^{\mathrm{e}}$ \\
\hline Type $I^{c}$ & $-0.614(0.001)^{\mathrm{e}}$ & $-0.622[-0.809,-0.298]^{\mathrm{e}}$ & $-2.21(-3.38,-1.04)^{\mathrm{e}}$ & $0.001^{e}$ & $0.35^{\mathrm{e}}$ & $-2.05(-3.13,-0.96)^{\mathrm{e}}$ & $0.001^{\mathrm{e}}$ & $0.45^{\mathrm{e}}$ \\
\hline Type III-IV & $-0.547(0.003)^{\mathrm{e}}$ & $-0.633[-0.816,-0.315]^{\mathrm{e}}$ & $-0.31(-0.50,-0.11)^{\mathrm{e}}$ & $0.003^{e}$ & $0.27^{\mathrm{e}}$ & $-0.32(-0.49,-0.16)^{\mathrm{e}}$ & $0.001^{e}$ & $0.47^{\mathrm{e}}$ \\
\hline
\end{tabular}

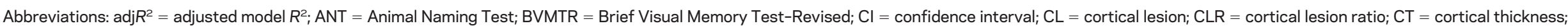

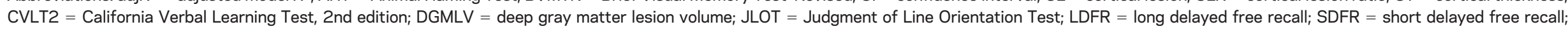
SDMT = Symbol Digit Modalities Test; WMLV = white matter lesion volume; WTAR = Wechsler Test of Adult Reading.

aUnivariate Pearson correlation

b Partial correlation coefficient.

${ }^{\mathrm{c}}$ Indicates square root-transformed variable.

${ }^{\mathrm{d}}$ Indicates log-transformed variable.

e Significant data. 
at the pial surface in association with diffuse or focal meningeal inflammation, giving rise to subpial tissue changes, which may be followed over time by involvement of deeper cortical layers (type I, II, and IV lesions), axonal degeneration, and increasing disability. ${ }^{34,35}$ Although our cross-sectional observations are not sufficient to draw firm conclusions regarding the "outsidein" hypothesis, it is clear that subpial lesions are present from the clinical onset of disease, as reported in biopsy sampling cases of early disease. ${ }^{34}$ This is a testable hypothesis through longitudinal in vivo imaging using 7T MRI that may help elucidate early pathophysiologic processes with implications for physical and cognitive deficits in MS.

There are several limitations to the presented data that require caution with interpretation. We did not specifically investigate deep gray matter atrophy and its contribution to cognitive performance; rather, we focused our analysis on cortical metrics. We did not correct for a multiplicity of statistical tests for the purpose of identifying testable hypotheses for future investigation. Seven patients $(21 \%)$ in our cohort produced suboptimal images for analysis due to motion artifact. Because of increased spatial resolution at 7T combined with multichannel radiofrequency technology, patient motion is exaggerated at the cortex, yielding degraded images. Although spatial resolution is excellent at ultra-high-field MRI, , ,9,28,36,37 type II CLs (the least frequent CL disclosed on histopathology inspection) remain a challenge to identify in vivo, presumably as a result of their small size relative to even this degree of resolution. In our cohort, type II CLs were exceedingly rare. Indeed, type II CLs smaller than $1.1 \mathrm{~mm}$ in diameter are less frequently detected at 7T. ${ }^{9}$ As such, the pathophysiologic role of type II CLs is elusive. Additionally, our global cortical and WM MRI metric analysis may be most sensitive to globally distributed cognitive domains, while being insensitive to regionally driven cognitive processes. An automated surface-based approach with both global and regional analyses of $\mathrm{T}^{*}$ signal change in the cortex may prove to be a more objective MRI metric at $7 \mathrm{~T}$ to quantify intracortical pathology for future study of cognitive dysfunction in MS. ${ }^{38}$

Our preliminary data identify CL metrics that are significantly associated with neurologic disability (primarily type III-IV CLs) and cognitive performance in MS - particularly the subpial (type III-IV) and leukocortical (type I) CLs that have not been associated previously in vivo. Moreover, we were able to determine these correlations with a small group of patients with MS. Because cognitive impairment in MS increases as the disease ensues, with or without similar levels of physical disability progression, further longitudinal observation of this cohort should allow us to identify global and regional mechanisms of injury responsible for both physical and cognitive disease progression. ${ }^{4,39}$

\section{AUTHOR CONTRIBUTIONS}

Dr. Nielsen contributed to study design, analysis and interpretation, and drafting and revising the manuscript. Dr. Kinkel contributed by conceptualizing and designing the study, interpretation of analyses, and revising the manuscript. Dr. Madigan contributed to study design, data interpretation, and revising the manuscript. Dr. Tinelli and Dr. Benner contributed in extraction and analysis of radiographic data and revising the manuscript. Dr. Mainero contributed to study design and conceptualization, analysis and interpretation, and revising the manuscript.

\section{STUDY FUNDING}

C. Mainero and R. Kinkel were funded by a grant from the National Multiple Sclerosis Society (NMSS 4281-RG-A1). A. Nielsen is funded through participation in the Harvard Medical School SCSP (NH 1 KL2 RR025757-0), and a Sylvia Lawry Physician Fellowship Award through the National Multiple Sclerosis Society (NMSS FP 1770A1).

\section{DISCLOSURE}

A. Nielsen received fellowship training and financial support through the NMSS (FP 1770A1) and Harvard Medical School SCSP (NH 1 KL2 RR025757-0). R. Kinkel is a scientific consultant for Biogen; serves as section editor of Neurology MedReviews; receives research support from Biogen Idec; receives partial salary support from Harvard Medical School; and receives support for his fellowship training program from the NMSS and support for research activities with the Accelerated Cure Project. N. Madigan and E. Tinelli report no disclosures. T. Benner served as a consultant for Siemens Medical Solutions and Bayer. C. Mainero served as a consultant for Biogen and receives research support from NMSS 4281-RG-A1 (PI). Go to Neurology.org for full disclosures.

Received October 3, 2012. Accepted in final form April 29, 2013.

\section{REFERENCES}

1. Rao S, Leo G, Bernardin L, Unverzagt F. Cognitive dysfunction in multiple sclerosis: frequency, patterns, and predictions. Neurology 1991;41:685-691.

2. Benedict R, Zivadinov R. Risk factors for and management of cognitive dysfunction in multiple sclerosis. Nat Rev Neurol 2011;7:332-342.

3. Rao S, Leo G, Ellington I, Nauertz T, Bernardin L, Unverzagt F. Cognitive dysfunction in multiple sclerosis. II. Impact on employment and social functioning. Neurology 1991;41:692-696.

4. Filippi M, Rocca M, Benedict R, et al. The contribution of MRI in assessing cognitive impairment in multiple sclerosis. Neurology 2010;75:2121-2128.

5. Roosendaal S, Moraal B, Pouwels P, et al. Accumulation of cortical lesions in MS: relation with cognitive impairment. Mult Scler 2009;15:708-714.

6. Calabrese M, Agosta F, Rinaldi F, et al. Cortical lesions and atrophy associated with cognitive impairment in relapsingremitting multiple sclerosis. Arch Neurol 2009;66:1144-1150.

7. Kutzelnigg A, Lucchinetti C, Stadelmann C, et al. Cortical demyelination and diffuse white matter injury in multiple sclerosis. Brain 2005;128:2705-2712.

8. Nielsen AS, Kinkel R, Tinelli E, Benner T, Cohen-Adad J, Mainero C. Focal cortical lesion detection in multiple sclerosis: 3 tesla DIR versus 7 tesla FLASH-T2*. J Magn Reson Imaging 2012;35:537-542.

9. Pitt D, Boster A, Pei W, et al. Imaging cortical lesions in multiple sclerosis with ultra-high-field magnetic resonance imaging. Arch Neurol 2010;67:812-818. 
10. Morrissey S, Miller D, Kendall B, et al. The significance of brain magnetic resonance imaging abnormalities at presentation with clinically isolated syndromes suggestive of multiple sclerosis: a 5-year follow-up study. Brain 1993;116(pt 1): 135-146.

11. Polman $\mathrm{CH}$, Reingold SC, Edan G, et al. Diagnostic criteria for multiple sclerosis: 2005 revisions to the "McDonald criteria." Ann Neurol 2005;58:840-846.

12. Kurtzke J. Rating neurologic impairment in multiple sclerosis: an expanded disability status scale (EDSS). Neurology 1983;33:1444-1452.

13. Delis D, Kramer J, Kaplan E, Ober B. California Verbal Learning Test (Adult Version Manual), 2nd ed. San Antonio: The Psychological Corporation; 2000.

14. Benedict R. Brief Visuospatial Memory Test-Revised. Odessa, FL: Psychological Assessment Resources; 1997.

15. Kongs S, Thompson L, Iverson G, Heaton R. Wisconsin Card Sorting Test-64 Card Version. Lutz, FL: Psychological Assessment Resources; 2000.

16. Smith A. Symbol Digit Modalities Test Manual-Revised. Los Angeles: Western Psychological Services; 1991.

17. Benton A, Sivan A, Hamsher K, Varney N, Spreen O. Contributions to Neuropsychological Assessment, 2nd ed. New York: Oxford University Press; 1994.

18. Tombaugh T, Kozak J, Rees L. Normative data stratified by age and education for two measures of verbal fluency: FAS and animal naming. Arch Clin Neuropsychol 1999; 14:167-177.

19. Benton A, Hamsher K. Multilingual Aphasia Examination. Iowa City: AJA Associates; 1989.

20. Wechsler D. Wechsler Test of Adult Reading (WTAR) Manual. San Antonio: The Psychological Corporation; 2001.

21. Beck A, Steer R, Brown O. Beck Depression Inventory, 2nd ed. San Antonio: The Psychological Corporation; 1996.

22. van der Kouwe A, Benner T, Fischl B, et al. On-line automatic slice positioning for brain MR imaging. Neuroimage 2005;27:222-230.

23. Peterson J, Bo L, Mork S, Chang A, Trapp B. Transected neurites, apoptotic neurons, and reduced inflammation in cortical multiple sclerosis lesions. Ann Neurol 2001;50: 389-400.

24. Bo L, Vedeler C, Nyland H, Trapp B, Mork S. Intracortical multiple sclerosis lesions are not associated with increased lymphocyte infiltration. Mult Scler 2003;9:323-331.

25. Vercellino M, Plano F, Votta B, Mutani R, Biordana M, Cavalla P. Grey matter pathology in multiple sclerosis. J Neuropathol Exp Neurol 2005;64:1101-1107.
26. Dale A, Fischl B, Sereno M. Cortical surface-based analysis. I. Segmentation and surface reconstruction. Neuroimage 1999;9:179-194.

27. Fischl B, Liu A, Dale A. Automated manifold surgery: constructing geometrically accurate and topologically correct models of the human cerebral cortex. IEEE Trans Med Imaging 2001;20:70-80.

28. Mainero C, Benner T, Radding A, et al. In vivo imaging of cortical pathology in multiple sclerosis using ultra-high field MRI. Neurology 2009;73:941-948.

29. Magliozzi R, Howell O, Vora A, et al. Meningeal B-cell follicles in secondary progressive multiple sclerosis associate with early onset of disease and severe cortical pathology. Brain 2007;130(pt 4):1089-1104.

30. Mike A, Glanz B, Hildenbrand P, et al. Identification and clinical impact of multiple sclerosis cortical lesions as assessed by routine 3T MR imaging. Am J Neuroradiol 2011;32:515-521.

31. Geurts J, Roosendaal S, Calabrese M, et al. Consensus recommendations for MS cortical lesion scoring using double inversion recovery MRI. Neurology 2011;76:418-424.

32. Hulst E, Steenwijk D, Versteeg A, et al. Cognitive impairment in MS: impact of white matter integrity, gray matter volume, and lesions. Neurology 2013;80:1025-1032.

33. Nelson F, Datta S, Garcia N, et al. Intracortical lesions by $3 \mathrm{~T}$ magnetic resonance imaging and correlation with cognitive impairment in multiple sclerosis. Mult Scler 2011; 17:1122-1129.

34. Lucchinetti C, Popescu B, Bunyan R, et al. Inflammatory cortical demyelination in early multiple sclerosis. N Engl J Med 2011;365:2188-2197.

35. Geurts J, Stys P, Minagar A, Amor S, Zivadinov R. Gray matter pathology in (chronic) MS: modern views on an early observation. J Neurol Sci 2009;282:12-20.

36. Vaughan J, Garwood M, Collins C, et al. 7T vs. 4T: RF power, homogeneity, and signal-to-noise comparison in head images. Magn Reson Med 2001;46:24-30.

37. Wiggins G, Potthast A, Triantafyllou C, Wiggins C, Wald L. Eight-channel phased array coil and detunable TEM volume coil for 7T brain imaging. Magn Reson Med 2005;54: 235-240.

38. Cohen-Adad J, Benner T, Greve D, et al. In vivo evidence of disseminated subpial $\mathrm{T}^{*}$ signal changes in multiple sclerosis at 7T: a surface-based analysis. Neuroimage 2011;57:55-62.

39. Reuter F, Zaaraoui W, Crespy L, et al. Frequency of cognitive impairment dramatically increases during the first 5 years of multiple sclerosis. J Neurol Neurosurg Psychiatry 2011;82:1157-1159. 